\title{
Editorial: Scaffolds for Regenerative Medicine: A Special Issue of the Annals of Biomedical Engineering
}

The field of tissue engineering and regenerative medicine (TE/RM) still faces considerable scientific, technical and regulatory challenges in translating its approaches from bench to bedside. But the multiple benefits of replacing tissues and organs lost to disease or trauma are tantalizingly clear and, given recent exciting advances in the field, may be within our grasp.

Design of an engineered tissue intended to become a functional transplant has historically depended on integration of three different scientific thrusts: relevant cell type(s), a biocompatible supporting structure, and bioactive molecules. Recent advances in understanding and controlling cell biology and development have focused much attention on the control of cell fate, mostly via timely application of specific growth factors. Yet, this emerging understanding of how cells relate to each other and their local environment demands a synthesis of our growing understanding of that environment. Bioactive scaffolds are widely regarded as key players in functional maturation of engineered tissue constructs as well as in tissue regeneration in situ, that can accomplish a plethora of different functions including guiding cellular and tissue responses, controlling cell migration and homing, delivering bioactive molecules and providing structural support and architecture for developing tissues. This special issue of the Annals of Biomedical Engineering was conceived as a vehicle to address recent progress in scaffold design and to serve as a resource for the community with respect to the current state-of-the-art in the field.

The reviews have been loosely organized around four broad themes: "building blocks and physical properties", "cell-host interactions", "assembling the parts", and "perspectives on translation". The editors recruited expertise across a spectrum of areas from the basic elements (cells, biomaterials, and bioactive molecules), to tools and approaches for directing and guiding tissue assembly and monitoring the tissue

The editors all contributed equally to the assembling of the special issue and the introductory editorial. The views expressed herein are those of the authors and do not necessarily represent those of the National Institutes of Health or the US Government.

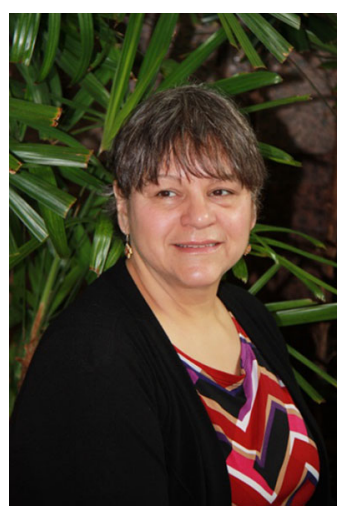

Rosemarie Hunziker, PhD, Director, Tissue Engineering/ Regenerative Medicine and Biomaterials Programs, National Institute of Biomedical Imaging and Bioengineering (NIBIB), National Institutes of Health (NIH).

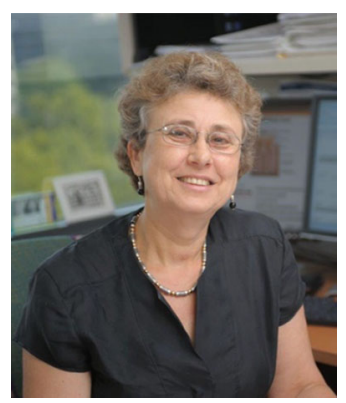

Nadya Lumelsky, PhD, Director, Tissue Engineering and Regenerative Medicine Program, National Institute of Dental and Craniofacial Research (NIDCR), National Institutes of Health (NIH).

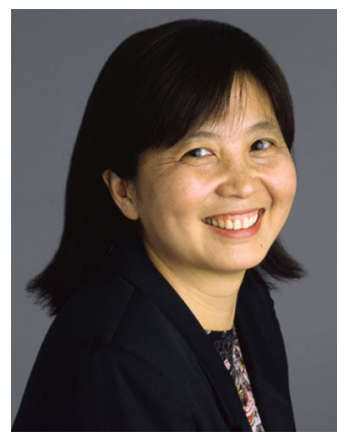

Fei Wang, PhD, Director, Musculoskeletal Tissue Engineering and Regenerative Medicine Program, National Institute of Arthritis and Musculoskeletal and Skin Diseases (NIAMS), National Institutes of Health (NIH). 
regeneration, to functional integration between engineered and host tissues and finally to challenges facing clinical deployment of TE/RM technologies. Additional topics, such as the role of immune cells in tissue vascularization and healing, pros and cons of natural vs synthetic materials as scaffolds, have also been highlighted. Some reviews favor the idea that cells alone should serve as a starting material for new tissues and that these cells would be able to synthesize their own appropriate matrices to support their functional maturation. Others propose a more traditional approach of designing bioactive scaffolds that can recruit cells, and guide their differentiation and tissue morphogenesis. While a diversity of tissues and organs are covered in the special issue, musculoskeletal tissues occupy the central place, as reflective of the collective research in the field at this time.

While recent years brought a tremendous progress to $\mathrm{TE} / \mathrm{RM}$ as a whole, many unresolved issues and questions remain. The editors certainly hope that this volume will serve as a catalyst that will prompt practitioners in the field to ask and solve difficult questions, and to bring much-needed TE/RM therapies to clinic.

\section{Rosemarie HuNZIKer}

Tissue Engineering/Regenerative Medicine and Biomaterials Programs, National Institute of Biomedical Imaging and Bioengineering (NIBIB), National Institutes of Health (NIH),

Bethesda, MD, USA

Electronic mail: hunzikerr@mail.nih.gov

\section{NADYA LUMELSKY}

Tissue Engineering and Regenerative Medicine Program, National Institute of Dental and Craniofacial Research (NIDCR), National Institutes of Health (NIH), Bethesda, MD, USA

FEI WANG

Musculoskeletal Tissue Engineering and Regenerative Medicine Program, National Institute of Arthritis and Musculoskeletal and Skin Diseases (NIAMS), National Institutes of Health (NIH), Bethesda, MD, USA 